\title{
On Weighted Montgomery Identity for $k$ Points and Its Associates on Time Scales
}

\author{
Eze R. Nwaeze and Ana M. Tameru \\ Department of Mathematics, Tuskegee University, Tuskegee, AL 36088, USA \\ Correspondence should be addressed to Eze R. Nwaeze; enwaeze@mytu.tuskegee.edu
}

Received 21 March 2017; Accepted 6 June 2017; Published 30 July 2017

Academic Editor: Patricia J. Y. Wong

Copyright (C) 2017 Eze R. Nwaeze and Ana M. Tameru. This is an open access article distributed under the Creative Commons Attribution License, which permits unrestricted use, distribution, and reproduction in any medium, provided the original work is properly cited.

\begin{abstract}
The purpose of this paper is to establish a weighted Montgomery identity for $k$ points and then use this identity to prove a new weighted Ostrowski type inequality. Our results boil down to the results of Liu and Ngô if we take the weight function to be the identity map. In addition, we also generalize an inequality of Ostrowski-Grüss type on time scales for $k$ points. For $k=2$, we recapture a result of Tuna and Daghan. Finally, we apply our results to the continuous, discrete, and quantum calculus to obtain more results in this direction.
\end{abstract}

\section{Introduction}

In 1938, Ostrowski [1] proved the following inequality which approximates a function by its integral average.

Theorem 1. Let $f:[a, b] \rightarrow \mathbb{R}$ be a differentiable mapping on $(a, b)$ with the property that $\left|f^{\prime}(x)\right| \leq M$ for all $x \in(a, b)$. Then

$$
\begin{aligned}
\left|f(x)-\frac{1}{b-a} \int_{a}^{b} f(t) d t\right| \\
\quad \leq\left[\frac{1}{4}+\frac{(x-(a+b) / 2)^{2}}{(b-a)^{2}}\right](b-a) M,
\end{aligned}
$$

for all $x \in[a, b]$. The constant $1 / 4$ is the best possible in the sense that it cannot be replaced by a smaller constant.

In 1997, Dragomir and Wang [2] obtained another inequality of this type.

Theorem 2. If $f:[a, b] \rightarrow \mathbb{R}$ is differentiable on $[a, b]$ and $\gamma \leq f^{\prime}(x) \leq \Gamma$, for all $x \in[a, b]$ for some constants $\gamma, \Gamma \in \mathbb{R}$, then

$$
f(x)-\frac{1}{b-a} \int_{a}^{b} f(t) d t
$$

$$
-\frac{f(b)-f(a)}{b-a}\left(x-\frac{a+b}{2}\right) \mid \leq \frac{1}{4}(b-a)(\Gamma-\gamma),
$$

for all $x \in[a, b]$.

In 1988, the German Mathematician Hilger [3] introduced the concept of time scales. The time scale calculus is a unification of the theory of difference equations with that of differential equations, unifying integral and differential calculus with the calculus of finite differences, offering formalism for studying hybrid discrete-continuous dynamical system. Since the introduction of this theory, it became a point of research to extend known classical differential and integral results to time scales. Following this line of thought, Bohner and Matthews [4] extended Theorem 1 to time scales by proving the following result.

Theorem 3. Let $a, b, x, t \in \mathbb{T}, a<b$, and $f:[a, b] \rightarrow \mathbb{R}$ be differentiable. Then, for all $x \in[a, b]$, one has

$$
\begin{aligned}
& \left|f(x)-\frac{1}{b-a} \int_{a}^{b} f^{\sigma}(t) \Delta t\right| \\
& \leq \frac{M}{b-a}\left(h_{2}(x, a)+h_{2}(x, b)\right),
\end{aligned}
$$


where $h_{2}(\cdot, \cdot)$ is given in Definition 12 and $M=$ $\sup _{a<t<b}\left|f^{\Delta}(t)\right|<\infty$. This inequality is sharp in the sense that the right-hand side of (3) cannot be replaced by a smaller one.

For more generalizations, extensions, and variants of Theorem 3, we refer the interested reader to papers [5-10] and the references therein. In 2008, Liu and Ngô [11] generalized Theorem 3 for $k$ points $x_{1}, x_{2}, \ldots, x_{k}$. Specifically, they proved the following theorem.

Theorem 4. Suppose that

(1) $a, b \in \mathbb{T}, I_{k}: a=x_{0}<x_{1}<\cdots<x_{k-1}<x_{k}=b$ is $a$ division of the interval $[a, b]$ for $x_{0}, x_{1}, \ldots, x_{k} \in \mathbb{T}$,

(2) $\alpha_{j} \in \mathbb{T}(j=0,1, \ldots, k+1)$ is $k+2$ points so that $\alpha_{0}=a, \alpha_{j} \in\left[x_{j-1}, x_{j}\right](j=1, \ldots, k)$ and $\alpha_{k+1}=b$,

(3) $f:[a, b] \rightarrow \mathbb{R}$ is differentiable function.

Then one has

$$
\begin{aligned}
& \left|\int_{a}^{b} f^{\sigma}(t) \Delta t-\sum_{j=0}^{k}\left(\alpha_{j+1}-\alpha_{j}\right) f\left(x_{j}\right)\right| \\
& \quad \leq M \sum_{j=0}^{k-1}\left(h_{2}\left(x_{j}, \alpha_{j+1}\right)+h_{2}\left(x_{j+1}, \alpha_{j+1}\right)\right),
\end{aligned}
$$

where $M=\sup _{a<t<b}\left|f^{\Delta}(t)\right|$. This inequality is sharp in the sense that the right-hand side of (4) cannot be replaced by a smaller one.

As a consequence (by taking $\lambda=0$ ) of Theorem 9 in [12], Tuna and Daghan obtained the following time scale version of Theorem 2.

Theorem 5. Let $a, b, x, t \in \mathbb{T}, a<b$, and $f:[a, b] \rightarrow \mathbb{R}$ be differentiable. If $f^{\Delta}$ is rd-continuous and $\gamma \leq f^{\Delta}(t) \leq \Gamma$, for all $t \in[a, b]$ and for some $\gamma, \Gamma \in \mathbb{R}$, then, for all $x \in[a, b]$, one has

$$
\begin{aligned}
& \mid f(x)-\frac{1}{b-a} \int_{a}^{b} f^{\sigma}(t) \Delta t \\
& \quad-\frac{\Gamma+\gamma}{2(b-a)}\left[h_{2}(x, a)-h_{2}(x, b)\right] \mid \\
& \quad \leq \frac{\Gamma-\gamma}{2(b-a)}\left[h_{2}(x, a)+h_{2}(x, b)\right] .
\end{aligned}
$$

The aim of this paper is twofold, namely,

(1) proving a generalized weighted Montgomery identity for $k$ points $x_{1}, x_{2}, \ldots, x_{k}$. Using this identity, we then obtain a weighted version of Theorem 4 . Our result boils down to Theorem 4, if the weight function is the identity map,

(2) generalizing Theorem 5 for $k$ points $x_{1}, x_{2}, \ldots, x_{k}$. For the case where $k=2$, we recover Theorem 5 .
This present paper is organized as follows. In Section 2, we provide some time scale essentials that will aid in better understanding of what follows. Our main results are then stated and proven in Section 3. Finally, we apply our results to the continuous, discrete, and quantum calculus to obtain more results in this direction. A brief conclusion follows thereafter in Section 4.

\section{Preliminaries}

Now, we briefly introduce the theory of time scales. For an in-depth study of the time scale calculus, we recommend the books of Bohner and Peterson [13, 14].

Definition 6. A time scale $\mathbb{T}$ is an arbitrary nonempty closed subset of $\mathbb{R}$. The forward jump operator $\sigma: \mathbb{T} \rightarrow \mathbb{T}$ and backward jump operator $\rho: \mathbb{T} \rightarrow \mathbb{T}$ are defined by $\sigma(t):=$ $\inf \{s \in \mathbb{T}: s>t\}$ for $t \in \mathbb{T}$ and $\rho(t):=\sup \{s \in \mathbb{T}: s<t\}$ for $t \in \mathbb{T}$, respectively. Clearly, we see that $\sigma(t) \geq t$ and $\rho(t) \leq t$ for all $t \in \mathbb{T}$. If $\sigma(t)>t$, then we say that $t$ is right-scattered, while if $\rho(t)<t$, then we say that $t$ is left-scattered. If $\sigma(t)=t$, then $t$ is called right dense, and if $\rho(t)=t$, then $t$ is called left dense. Points that are both right dense and left dense are called dense. The set $\mathbb{T}^{k}$ is defined as follows: if $\mathbb{T}$ has a leftscattered maximum $m$, then $\mathbb{T}^{k}=\mathbb{T}-m$; otherwise, $\mathbb{T}^{k}=\mathbb{T}$. For $a, b \in \mathbb{T}$ with $a \leq b$, we define the interval $[a, b]$ in $\mathbb{T}$ by $[a, b]=\{t \in \mathbb{T}: a \leq t \leq b\}$. Open intervals and half-open intervals are defined in the same manner.

Definition 7. The function $f: \mathbb{T} \rightarrow \mathbb{R}$, is called differentiable at $t \in \mathbb{T}^{k}$, with delta derivative $f^{\Delta}(t) \in \mathbb{R}$, if for any given $\epsilon>0$ there exists a neighborhood $U$ of $t$ such that

$$
\left|f(\sigma(t))-f(s)-f^{\Delta}(t)(\sigma(t)-s)\right| \leq \epsilon|\sigma(t)-s|,
$$

$\forall s \in U$.

If $\mathbb{T}=\mathbb{R}$, then $f^{\Delta}(t)=d f(t) / d t$, and if $\mathbb{T}=\mathbb{Z}$, then $f^{\Delta}(t)=f(t+1)-f(t)$.

Definition 8. The function $f: \mathbb{T} \rightarrow \mathbb{R}$ is said to be $r d$ continuous if it is continuous at all right-dense points $t \in \mathbb{T}$ and its left-sided limits exist at all left-dense points $t \in \mathbb{T}$.

Definition 9. Let $f$ be $r d$-continuous function. Then $g: \mathbb{T} \rightarrow$ $\mathbb{R}$ is called an antiderivative of $f$ on $\mathbb{T}$ if it is differentiable on $\mathbb{T}$ and satisfies $g^{\Delta}(t)=f(t)$ for any $t \in \mathbb{T}^{k}$. In this case, one has

$$
\int_{a}^{b} f(s) \Delta s=g(b)-g(a) .
$$

Definition 10. The function $f^{\sigma}: \mathbb{T} \rightarrow \mathbb{R}$ is defined as

$$
f^{\sigma}(t):=f(\sigma(t))
$$

for any $t \in \mathbb{T}$.

Theorem 11. If $a, b, c \in \mathbb{T}$ with $a<c<b, \alpha \in \mathbb{R}$, and $f, g$ are rd-continuous, then one has the following: 
(i) $\int_{a}^{b}[f(t)+g(t)] \Delta t=\int_{a}^{b} f(t) \Delta t+\int_{a}^{b} g(t) \Delta t$.

(ii) $\int_{a}^{b} \alpha f(t) \Delta t=\alpha \int_{a}^{b} f(t) \Delta t$.

(iii) $\int_{a}^{b} f(t) \Delta t=-\int_{b}^{a} f(t) \Delta t$.

(iv) $\int_{a}^{b} f(t) \Delta t=\int_{a}^{c} f(t) \Delta t+\int_{c}^{b} f(t) \Delta t$.

(v) $\left|\int_{a}^{b} f(t) \Delta t\right| \leq \int_{a}^{b}|f(t)| \Delta t$.

(vi) $\int_{a}^{b} f(t) g^{\Delta}(t) \Delta t=(f g)(b)-(f g)(a)-\int_{a}^{b} f^{\Delta}(t) g^{\sigma}(t) \Delta t$.

Definition 12. Let $h_{k}: \mathbb{T}^{2} \rightarrow \mathbb{R}, k \in \mathbb{N}$, be functions that are recursively defined as

$$
\begin{aligned}
h_{0}(t, s) & =1, \\
h_{k+1}(t, s) & =\int_{s}^{t} h_{k}(\tau, s) \Delta \tau,
\end{aligned}
$$

$\forall s, t \in \mathbb{T}$.

\section{Main Results}

For the proof of our theorems, we will need the following lemma.

Lemma 13 (generalized weighted Montgomery identity for $k$ points). Suppose that

(1) $a, b \in \mathbb{T}, I_{k}: a=x_{0}<x_{1}<\cdots<x_{k-1}<x_{k}=b$ is $a$ division of the interval $[a, b]$ for $x_{0}, x_{1}, \ldots, x_{k} \in \mathbb{T}$,

(2) $\alpha_{j} \in \mathbb{T}(j=0,1, \ldots, k+1)$ is $k+2$ points so that $\alpha_{0}=a, \alpha_{j} \in\left[x_{j-1}, x_{j}\right](j=1, \ldots, k)$ and $\alpha_{k+1}=b$,

(3) $f, w:[a, b] \rightarrow \mathbb{R}$ are differentiable functions.

Then one has the following equation:

$$
\begin{aligned}
\int_{a}^{b} K\left(t, I_{k}\right) f^{\Delta}(t) \Delta t+\int_{a}^{b} w^{\Delta}(t) f^{\sigma}(t) \Delta t \\
=\left(\alpha_{1}-w(a)\right) f(a)+\left(w(b)-\alpha_{k}\right) f(b) \\
\quad+\sum_{j=1}^{k-1}\left(\alpha_{j+1}-\alpha_{j}\right) f\left(x_{j}\right),
\end{aligned}
$$

where

$$
K\left(t, I_{k}\right)= \begin{cases}w(t)-\alpha_{1}, & t \in\left[a, x_{1}\right), \\ w(t)-\alpha_{2}, & t \in\left[x_{1}, x_{2}\right), \\ \vdots & \\ w(t)-\alpha_{k-1}, & t \in\left[x_{k-2}, x_{k-1}\right), \\ w(t)-\alpha_{k}, & t \in\left[x_{k-1}, b\right] .\end{cases}
$$

Proof. Using items (i), (ii), (iv), and (vi) of Theorem 11, we have

$$
\begin{array}{rl}
\int_{a}^{b} & K\left(t, I_{k}\right) f^{\Delta}(t) \Delta t=\sum_{j=0}^{k-1} \int_{x_{j}}^{x_{j+1}} K\left(t, I_{k}\right) f^{\Delta}(t) \Delta t \\
& =\sum_{j=0}^{k-1} \int_{x_{j}}^{x_{j+1}}\left(w(t)-\alpha_{j+1}\right) f^{\Delta}(t) \Delta t \\
& =\sum_{j=0}^{k-1}\left[\left(w\left(x_{j+1}\right)-\alpha_{j+1}\right) f\left(x_{j+1}\right)\right. \\
& \left.-\left(w\left(x_{j}\right)-\alpha_{j+1}\right) f\left(x_{j}\right)-\int_{x_{j}}^{x_{j+1}} w^{\Delta}(t) f^{\sigma}(t) \Delta t\right] \\
& =\sum_{j=0}^{k-1}\left[\left(\alpha_{j+1}-w\left(x_{j}\right)\right) f\left(x_{j}\right)\right. \\
+ & \left(w\left(x_{j+1}\right)-\alpha_{j+1}\right) f\left(x_{j+1}\right) \\
& \left.-\int_{x_{j}}^{x_{j+1}} w^{\Delta}(t) f^{\sigma}(t) \Delta t\right]=\left(\alpha_{1}-w(a)\right) f(a) \\
+ & \left(w(b)-\alpha_{k}\right) f(b)-\int_{a}^{b} w^{\Delta}(t) f^{\sigma}(t) \Delta t \\
& \left.+\alpha_{k}\right) f(b)-\int_{a}^{b} w^{\Delta}(t) f^{\sigma}(t) \Delta t+\sum_{j=1}^{k-1}\left(\alpha_{j+1}-\alpha_{j}\right) \\
+ & \sum_{j=1}^{k-1}\left(\alpha_{j+1}-w\left(x_{j}\right)\right) f\left(x_{j}\right)+\sum_{j=0}^{k-2}\left(w\left(x_{j+1}\right)\right. \\
- & \left.\alpha_{j+1}\right) f\left(x_{j+1}\right)=\left(\alpha_{1}-w(a)\right) f(a)+(w(b)
\end{array}
$$

Hence, the result follows.

Remark 14. The above lemma becomes Lemma 1 in paper [11] if we take $w(t)=t$.

Corollary 15. If $w(t)=t^{2}$, then $w^{\Delta}(t)=\sigma(t)+t$ and (10) boils down to

$$
\begin{aligned}
\int_{a}^{b} K\left(t, I_{k}\right) f^{\Delta}(t) \Delta t+\int_{a}^{b}(\sigma(t)+t) f^{\sigma}(t) \Delta t \\
=\left(\alpha_{1}-a^{2}\right) f(a)+\left(b^{2}-\alpha_{k}\right) f(b) \\
\quad+\sum_{j=1}^{k-1}\left(\alpha_{j+1}-\alpha_{j}\right) f\left(x_{j}\right),
\end{aligned}
$$

where

$$
K\left(t, I_{k}\right)= \begin{cases}t^{2}-\alpha_{1}, & t \in\left[a, x_{1}\right), \\ t^{2}-\alpha_{2}, & t \in\left[x_{1}, x_{2}\right), \\ \vdots & \\ t^{2}-\alpha_{k-1}, & t \in\left[x_{k-2}, x_{k-1}\right), \\ t^{2}-\alpha_{k}, & t \in\left[x_{k-1}, b\right] .\end{cases}
$$


Corollary 16. If we take $\mathbb{T}=\mathbb{R}$ in Corollary 15, (13) becomes

$$
\begin{gathered}
\int_{a}^{b} K\left(t, I_{k}\right) f^{\prime}(t) d t+2 \int_{a}^{b} t f(t) d t \\
=\left(\alpha_{1}-a^{2}\right) f(a)+\left(b^{2}-\alpha_{k}\right) f(b) \\
+\sum_{j=1}^{k-1}\left(\alpha_{j+1}-\alpha_{j}\right) f\left(x_{j}\right) .
\end{gathered}
$$

Theorem 17 (weighted Ostrowski type inequality for $k$ points). Under the assumptions of Lemma 13, one has the following inequality:

$$
\begin{aligned}
& \mid\left(\alpha_{1}-w(a)\right) f(a)+\left(w(b)-\alpha_{k}\right) f(b) \\
& \quad+\sum_{j=1}^{k-1}\left(\alpha_{j+1}-\alpha_{j}\right) f\left(x_{j}\right)-\int_{a}^{b} w^{\Delta}(t) f^{\sigma}(t) \Delta t \mid \\
& \quad \leq M \int_{a}^{b}\left|K\left(t, I_{k}\right)\right| \Delta t
\end{aligned}
$$

where $M=\sup _{a<t<b}\left|f^{\Delta}(t)\right|$.

Proof. The proof of inequality (16) follows by taking the absolute value of both sides of (10) and then applying item (v) of Theorem 11.

Remark 18. By choosing $w(t)=t$, we recapture Theorem 4 .

Corollary 19 (continuous case). Let $\mathbb{T}=\mathbb{R}$. Then, one has from (16) the following inequality:

$$
\begin{aligned}
& \mid\left(\alpha_{1}-w(a)\right) f(a)+\left(w(b)-\alpha_{k}\right) f(b) \\
& \quad+\sum_{j=1}^{k-1}\left(\alpha_{j+1}-\alpha_{j}\right) f\left(x_{j}\right)-\int_{a}^{b} w^{\prime}(t) f(t) d t \mid \\
& \quad \leq M \int_{a}^{b}\left|K\left(t, I_{k}\right)\right| d t,
\end{aligned}
$$

where $M=\sup _{a<t<b}\left|f^{\prime}(t)\right|$ and $K\left(t, I_{k}\right)$ is given by (11).

Corollary 20 (discrete case). Let $\mathbb{T}=\mathbb{Z}, a=0, b=n$. Suppose that

(1) $I_{k}: 0=j_{0}<j_{1}<\cdots<j_{k-1}<j_{k}=n$ is a division of $[0, n] \cap \mathbb{Z}$ for $j_{0}, j_{1}, \ldots, j_{k} \in \mathbb{Z}$

(2) $p_{i} \in \mathbb{Z}(i=0, \ldots, k+1)$ is $k+2$ points so that $p_{0}=$ $0, p_{i} \in\left[j_{i-1}, j_{i}\right] \cap \mathbb{Z}(i=1, \ldots, k)$ and $p_{k+1}=n$;

(3) $f(k)=x_{k}$.

Then, for any differentiable function $w:[0, n] \cap \mathbb{Z} \rightarrow \mathbb{R}$, and each $k=1,2, \ldots, n$, one has

$$
\mid\left(p_{1}-w(0)\right) x_{0}+\left(w(n)-p_{k}\right) x_{n}+\sum_{i=1}^{k-1}\left(p_{i+1}-p_{i}\right) x_{j_{i}}
$$

$$
-\sum_{j=1}^{n}(w(j)-w(j-1)) x_{j}\left|\leq M \sum_{j=0}^{n-1}\right| K\left(j, I_{k}\right) \mid,
$$

where $M=\sup _{i=1, \ldots, n-1}\left|\Delta x_{i}\right|$ and

$$
K\left(j, I_{k}\right)= \begin{cases}w(j)-p_{1}, & j \in\left[0, j_{1}\right), \\ w(j)-p_{2}, & j \in\left[j_{1}, j_{2}\right), \\ \vdots & \\ w(j)-p_{k-1}, & j \in\left[j_{k-2}, j_{k-1}\right), \\ w(j)-p_{k}, & j \in\left[j_{k-1}, n\right] .\end{cases}
$$
rem 5 .

Next, we formulate and prove a generalization of Theo-

Theorem 21 (generalized Ostrowski-Grüss type inequality for $k$ points). Suppose that

(1) $a, b \in \mathbb{T}, I_{k}: a=x_{0}<x_{1}<\cdots<x_{k-1}<x_{k}=b$ is $a$ division of the interval $[a, b]$ for $x_{0}, x_{1}, \ldots, x_{k} \in \mathbb{T}$,

(2) $\alpha_{j} \in \mathbb{T}(j=0,1, \ldots, k+1)$ is $k+2$ points so that $\alpha_{0}=a, \alpha_{j} \in\left[x_{j-1}, x_{j}\right](j=1, \ldots, k)$ and $\alpha_{k+1}=b$,

(3) $f:[a, b] \rightarrow \mathbb{R}$ is differentiable, $f^{\Delta}$ is $r d$-continuous, and there exist $\gamma, \Gamma \in \mathbb{R}$ such that $\gamma \leq f^{\Delta}(t) \leq \Gamma$ for all $t \in[a, b]$.

Then one has the following inequality:

$$
\begin{aligned}
& \mid \sum_{j=0}^{k}\left(\alpha_{j+1}-\alpha_{j}\right) f\left(x_{j}\right)-\int_{a}^{b} f^{\sigma}(t) \Delta t \\
& -\frac{\Gamma+\gamma}{2} \sum_{j=0}^{k-1}\left(h_{2}\left(x_{j+1}, \alpha_{j+1}\right)-h_{2}\left(x_{j}, \alpha_{j+1}\right)\right) \mid \\
& \quad \leq \frac{\Gamma-\gamma}{2} \sum_{j=0}^{k-1}\left(h_{2}\left(x_{j}, \alpha_{j+1}\right)+h_{2}\left(x_{j+1}, \alpha_{j+1}\right)\right) .
\end{aligned}
$$

Inequality (20) is sharp in the sense that the constant 1/2 on the right-hand side cannot be replaced by a smaller one.

Proof. Using Lemma 13 with $w(t)=t$, we obtain (see also [11, Lemma 1])

$$
\begin{aligned}
\int_{a}^{b} K\left(t, I_{k}\right) f^{\Delta}(t) \Delta t= & \sum_{j=0}^{k}\left(\alpha_{j+1}-\alpha_{j}\right) f\left(x_{j}\right) \\
& -\int_{a}^{b} f^{\sigma}(t) \Delta t
\end{aligned}
$$


where

$$
K\left(t, I_{k}\right)= \begin{cases}t-\alpha_{1}, & t \in\left[a, x_{1}\right), \\ t-\alpha_{2}, & t \in\left[x_{1}, x_{2}\right), \\ \vdots & \\ t-\alpha_{k-1}, & t \in\left[x_{k-2}, x_{k-1}\right), \\ t-\alpha_{k}, & t \in\left[x_{k-1}, b\right] .\end{cases}
$$

Also, from (22), we get

$$
\begin{array}{rl}
\int_{a}^{b} & K\left(t, I_{k}\right) \Delta t=\sum_{j=0}^{k-1} \int_{x_{j}}^{x_{j+1}}\left(t-\alpha_{j+1}\right) \Delta t \\
& =\sum_{j=0}^{k-1}\left[\int_{x_{j}}^{\alpha_{j+1}}\left(t-\alpha_{j+1}\right) \Delta t+\int_{\alpha_{j+1}}^{x_{j+1}}\left(t-\alpha_{j+1}\right) \Delta t\right] \\
& =\sum_{j=0}^{k-1}\left[\int_{\alpha_{j+1}}^{x_{j+1}}\left(t-\alpha_{j+1}\right) \Delta t-\int_{\alpha_{j+1}}^{x_{j}}\left(t-\alpha_{j+1}\right) \Delta t\right] \\
& =\sum_{j=0}^{k-1}\left(h_{2}\left(x_{j+1}, \alpha_{j+1}\right)-h_{2}\left(x_{j}, \alpha_{j+1}\right)\right) .
\end{array}
$$

Similarly, one gets

$$
\begin{aligned}
& \int_{a}^{b}\left|K\left(t, I_{k}\right)\right| \Delta t \\
& \quad=\sum_{j=0}^{k-1}\left(h_{2}\left(x_{j}, \alpha_{j+1}\right)+h_{2}\left(x_{j+1}, \alpha_{j+1}\right)\right) .
\end{aligned}
$$

Now, let $\Theta=(\Gamma+\gamma) / 2$. From assumption (3), $\gamma \leq f^{\Delta}(t) \leq \Gamma$ for all $t \in[a, b]$ implies that $\gamma-\Theta \leq f^{\Delta}(t)-\Theta \leq \Gamma-\Theta$ for all $t \in[a, b]$. This further implies that $\left|f^{\Delta}(t)-\Theta\right| \leq(\Gamma-\gamma) / 2$, for all $t \in[a, b]$. Hence,

$$
\max _{t \in[a, b]}\left|f^{\Delta}(t)-\Theta\right| \leq \frac{\Gamma-\gamma}{2} .
$$

Using (21) and (23), we obtain

$$
\begin{aligned}
& \int_{a}^{b} K\left(t, I_{k}\right)\left(f^{\Delta}(t)-\Theta\right) \Delta t \\
& =\sum_{j=0}^{k}\left(\alpha_{j+1}-\alpha_{j}\right) f\left(x_{j}\right)-\int_{a}^{b} f^{\sigma}(t) \Delta t \\
& \quad-\Theta \sum_{j=0}^{k-1}\left(h_{2}\left(x_{j+1}, \alpha_{j+1}\right)-h_{2}\left(x_{j}, \alpha_{j+1}\right)\right) .
\end{aligned}
$$

The left-hand side of (26) is estimated as follows:

$$
\begin{aligned}
& \left|\int_{a}^{b} K\left(t, I_{k}\right)\left(f^{\Delta}(t)-\Theta\right) \Delta t\right| \\
& \quad \leq \max _{t \in[a, b]}\left|f^{\Delta}(t)-\Theta\right| \int_{a}^{b}\left|K\left(t, I_{k}\right)\right| \Delta t .
\end{aligned}
$$

Using relation (25) in (27), we get

$$
\begin{gathered}
\left|\int_{a}^{b} K\left(t, I_{k}\right)\left(f^{\Delta}(t)-\Theta\right) \Delta t\right| \\
\leq \frac{\Gamma-\gamma}{2} \int_{a}^{b}\left|K\left(t, I_{k}\right)\right| \Delta t .
\end{gathered}
$$

Hence, the desired result follows.

Remark 22. If $x_{1}=x, \alpha_{0}=\alpha_{1}=a, \alpha_{2}=\alpha_{3}=x_{2}=b$, then Theorem 21 becomes Theorem 5 for the case where $k=2$.

We now apply Theorem 21 to different time scales.

Corollary 23 (continuous case). Taking $\mathbb{U}=\mathbb{R}$ in Theorem 21 amounts to the following inequality:

$$
\begin{aligned}
& \mid \sum_{j=0}^{k}\left(\alpha_{j+1}-\alpha_{j}\right) f\left(x_{j}\right)-\int_{a}^{b} f(t) d t \\
& -\frac{\Gamma+\gamma}{2}\left[\frac{b^{2}-a^{2}}{2}-\sum_{j=0}^{k-1} \alpha_{j+1}\left(x_{j+1}-x_{j}\right)\right] \mid \\
& \leq \frac{\Gamma-\gamma}{2}\left[\sum_{j=0}^{k-1}\left(\frac{x_{j+1}-x_{j}}{2}\right)^{2}\right. \\
& \left.+\sum_{j=0}^{k-1}\left(\alpha_{j+1}-\frac{x_{j+1}+x_{j}}{2}\right)^{2}\right] .
\end{aligned}
$$

Proof. In this case, $h_{2}(t, s)=(t-s)^{2} / 2$. Using this in inequality (20) gives the desired result.

Remark 24. The above corollary is the same as Corollary 2.1 in [15]. In other words, Theorem 21 extends Corollary 2.1, in paper [15], to time scales.

Corollary 25 (discrete case). Let $\mathbb{T}=\mathbb{Z}, a=0, b=n$. Suppose that

(1) $I_{k}: 0=j_{0}<j_{1}<\cdots<j_{k-1}<j_{k}=n$ is a division of $[0, n] \cap \mathbb{Z}$ for $j_{0}, j_{1}, \ldots, j_{k} \in \mathbb{Z}$

(2) $p_{i} \in \mathbb{Z}(i=0, \ldots, k+1)$ is $k+2$ points so that $p_{0}=$ $0, p_{i} \in\left[j_{i-1}, j_{i}\right] \cap \mathbb{Z}(i=1, \ldots, k)$ and $p_{k+1}=n$;

(3) $f(k)=x_{k}$.

Then one has the following inequality:

$$
\begin{aligned}
& \mid \sum_{i=0}^{k}\left(p_{i+1}-p_{i}\right) x_{j_{i}}-\sum_{j=1}^{n} x_{j} \\
& \quad+\frac{\Gamma+\gamma}{4}\left[n^{2}-\sum_{i=0}^{k-1}\left(\left(2 p_{i+1}-1\right)\left(j_{i+1}-j_{i}\right)\right)\right] \mid
\end{aligned}
$$




$$
\begin{aligned}
& \leq \frac{\Gamma-\gamma}{2}\left[\sum_{i=0}^{k-1}\left(\frac{j_{i+1}-j_{i}}{2}\right)^{2}\right. \\
& \left.+\sum_{i=0}^{k-1}\left(p_{i+1}-\frac{j_{i+1}+j_{i}}{2}\right)^{2}+\sum_{i=0}^{k-1}\left(p_{i+1}-\frac{j_{i+1}+j_{i}}{2}\right)\right] .
\end{aligned}
$$

Proof. The proof follows by using the inequality in Theorem 21 and observing that

$$
\begin{aligned}
h_{2}\left(j_{i}, p_{i+1}\right) & =\frac{\left(j_{i}-p_{i+1}\right)\left(j_{i}-p_{i+1}-1\right)}{2} \\
h_{2}\left(j_{i+1}, p_{i+1}\right) & =\frac{\left(j_{i+1}-p_{i+1}\right)\left(j_{i+1}-p_{i+1}-1\right)}{2} .
\end{aligned}
$$

Corollary 26 (quantum case). Let $\mathbb{T}=q^{\mathbb{N}_{0}}, q>1, a=$ $q^{m}, b=q^{n}$ with $m<n$. Suppose that

(1) $I_{k}: q^{m}=q^{j_{0}}<q^{j_{1}}<\cdots<q^{j_{k-1}}<q^{j_{k}}=q^{n}$ is a division of $\left[q^{m}, q^{n}\right] \cap q^{\mathbb{N}_{0}}$ for $j_{0}, j_{1}, \ldots, j_{k} \in \mathbb{N}_{0}$;

(2) $q^{p_{i}} \in q^{\mathbb{N}_{0}}(i=0, \ldots, k+1)$ is $k+2$ points so that $q^{p_{0}}=q^{m}, q^{p_{i}} \in\left[q^{j_{i-1}}, q^{j_{i}}\right] \cap q^{\mathbb{N}_{0}}(i=1, \ldots, k)$ and $q^{p_{k+1}}=q^{n}$

(3) $f:\left[q^{m}, q^{n}\right] \rightarrow \mathbb{R}$ is differentiable.

Then one has

$$
\begin{aligned}
& \mid \sum_{i=0}^{k}\left(q^{p_{i+1}}-q^{p_{i}}\right) f\left(q^{j_{i}}\right)-\int_{q^{m}}^{q^{n}} f(q t) d_{q} t-\frac{\Gamma+\gamma}{2(q+1)} \\
& \quad \cdot \sum_{i=0}^{k-1}\left[\left(q^{j_{i+1}}-q^{p_{i+1}}\right)\left(q^{j_{i+1}}-q^{p_{i+1}+1}\right)\right. \\
& \left.\quad-\left(q^{j_{i}}-q^{p_{i+1}}\right)\left(q^{j_{i}}-q^{p_{i+1}+1}\right)\right] \mid \leq \frac{\Gamma-\gamma}{2(q+1)} \\
& \quad \cdot \sum_{i=0}^{k-1}\left[\left(q^{j_{i+1}}-q^{p_{i+1}}\right)\left(q^{j_{i+1}}-q^{p_{i+1}+1}\right)+\left(q^{j_{i}}-q^{p_{i+1}}\right)\right. \\
& \left.\quad \cdot\left(q^{j_{i}}-q^{p_{i+1}+1}\right)\right] .
\end{aligned}
$$

Proof. Using Theorem 21 and the fact that, for the quantum calculus, one has $\sigma(t)=q t$,

$$
\begin{gathered}
h_{2}\left(q^{j_{i}}, q^{p_{i+1}}\right)=\frac{\left(q^{j_{i}}-q^{p_{i+1}}\right)\left(q^{j_{i}}-q^{p_{i+1}+1}\right)}{q+1}, \\
h_{2}\left(q^{j_{i+1}}, q^{p_{i+1}}\right)=\frac{\left(q^{j_{i+1}}-q^{p_{i+1}}\right)\left(q^{j_{i+1}}-q^{p_{i+1}+1}\right)}{q+1} .
\end{gathered}
$$

Corollary 27. Suppose that $\alpha \in[a, b] \cap \mathbb{T}$. Then one has the following inequality:

$$
\begin{aligned}
& \mid(\alpha-a) f(a)+(b-\alpha) f(b)-\int_{a}^{b} f^{\sigma}(t) \Delta t \\
& \quad-\frac{\Gamma+\gamma}{2}\left[h_{2}(b, \alpha)-h_{2}(a, \alpha)\right] \mid \leq \frac{\Gamma-\gamma}{2}\left[h_{2}(a, \alpha)\right. \\
& \left.\quad+h_{2}(b, \alpha)\right] .
\end{aligned}
$$

Proof. Inequality (34) follows by choosing $k=1, \alpha_{0}=$ $a, x_{1}=b, \alpha_{0}=a, \alpha_{1}=\alpha$, and $\alpha_{2}=b$, in Theorem 21 .

Remark 28. (1) Taking $\alpha=a$ in (34), we get

$$
\begin{aligned}
& \left|(b-a) f(b)-\int_{a}^{b} f^{\sigma}(t) \Delta t-\frac{\Gamma+\gamma}{2} h_{2}(b, a)\right| \\
& \leq \frac{\Gamma-\gamma}{2} h_{2}(b, a) .
\end{aligned}
$$

(2) Taking $\alpha=(a+b) / 2$ in (34), we get

$$
\begin{aligned}
& \mid \frac{f(a)+f(b)}{2}(b-a)-\int_{a}^{b} f^{\sigma}(t) \Delta t \\
& \quad-\frac{\Gamma+\gamma}{2}\left[h_{2}\left(b, \frac{a+b}{2}\right)-h_{2}\left(a, \frac{a+b}{2}\right)\right] \mid \\
& \quad \leq \frac{\Gamma-\gamma}{2}\left[h_{2}\left(a, \frac{a+b}{2}\right)+h_{2}\left(b, \frac{a+b}{2}\right)\right] .
\end{aligned}
$$

(3) Now, for $\alpha=b$ in (34), we obtain

$$
\begin{aligned}
& \left|(b-a) f(a)-\int_{a}^{b} f^{\sigma}(t) \Delta t+\frac{\Gamma+\gamma}{2} h_{2}(a, b)\right| \\
& \quad \leq \frac{\Gamma-\gamma}{2} h_{2}(a, b) .
\end{aligned}
$$

\section{Conclusion}

The Ostrowski and Ostrowski-Grüss inequalities have received great deal of attention from the mathematical community dealing with inequalities. Giant steps have been made in extending some of the results to time scales. This work is tailored towards advancing this move. To be precise, we proved a generalization of the Montgomery identity and then used the resultant equation to obtain a weighted Ostrowski inequality for $k$ points, thus generalizing a result of Liu and Ngô [11]. Furthermore, we obtained an OstrowskiGrüss type inequality which generalizes and extends results of Tuna and Daghan [12] and Feng and Meng [15].

\section{Conflicts of Interest}

The authors declare that there are no conflicts of interest regarding the publication of this paper. 


\section{References}

[1] S. S. Dragomir, "Grüss inequality in inner product spaces," Gazette of the Australian Mathematical Society, vol. 26, no. 2, pp. 66-70, 1999.

[2] S. S. Dragomir and S. Wang, "An inequality of Ostrowski-Grüss type and its applications to the estimation of error bounds for some special means and for some numerical quadrature rules," Computers and Mathematics with Applications, vol. 33, no. 11, pp. 16-20, 1997.

[3] S. Hilger, Ein Maßkettenkalkül mit Anwendung auf Zentrumsmannigfaltigkeiten [Ph.D. thesis], Universität Würzburg, Würzburg, Germany, 1988.

[4] M. Bohner and T. Matthews, "Ostrowski inequalities on time scales," Journal of Inequalities in Pure and Applied Mathematics, vol. 9, no. 1, article no. 6, 2008.

[5] B. Karpuz and U. M. Özkan, "Ostrowski Inequality on time scales," J. Inequal. Pure and Appl. Math, vol. 9, no. 4, article 112, 2008.

[6] W. Liu, A. Tuna, and Y. Jiang, "On weighted Ostrowski type, Trapezoid type, Grüss type and Ostrowski-Grüss like inequalities on time scales," Applicable Analysis, vol. 93, no. 3, pp. 551571, 2014.

[7] W. Liu, A. Tuna, and Y. Jiang, "New weighted Ostrowski and Ostrowski-Grüss type inequalities on time scales," Analele Stiintifice ale Universitatii Al I Cuza din Iasi - Matematica, vol. 60, no. 1, pp. 57-76, 2014.

[8] E. R. Nwaeze, "A new weighted Ostrowski type inequality on arbitrary time scale," Journal of King Saud University - Science, vol. 29, no. 2, pp. 230-234, 2017.

[9] E. R. Nwaeze, "Generalized weighted trapezoid and Grüss type inequalities on time scales," Australian Journal of Mathematical Analysis and Applications, vol. 11, no. 1, article 4, pp. 1-13, 2017.

[10] G. Xu and Z. B. Fang, "A Generalization of Ostrowski type inequality on time scales with $k$ points," Journal of Mathematical Inequalities, vol. 11, no. 1, pp. 41-48, 2017.

[11] W. Liu and Q.-A. Ngô, "A generalization of Ostrowski inequality on time scales for $k$ points," Applied Mathematics and Computation, vol. 203, no. 2, pp. 754-760, 2008.

[12] A. Tuna and D. Daghan, "Generalization of Ostrowski and Ostrowski-Grüss type inequalities on time scales," Computers and Mathematics with Applications, vol. 60, no. 3, pp. 803-811, 2010.

[13] M. Bohner and A. Peterson, Dynamic Equations on Time Scales: An Introduction with Applications, Birkhauser, Boston, Mass, USA, 2001.

[14] M. Bohner and A. Peterson, Advances in Dynamic Equations on Time Series, Birkhäuser, Boston, MA, USA, 2003.

[15] Q. Feng and F. Meng, "New Ostrowski-Grüss type inequalities with the derivatives bounded by functions," Journal of Inequalities and Applications, vol. 2013, article no. 456, 2013. 


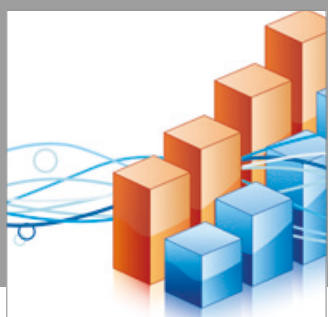

Advances in

Operations Research

vatersals

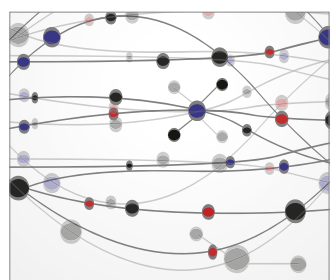

\section{The Scientific} World Journal
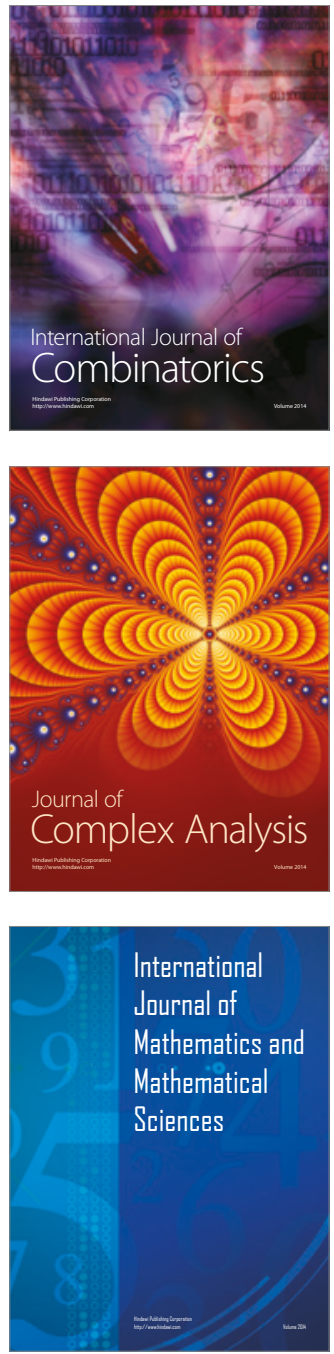
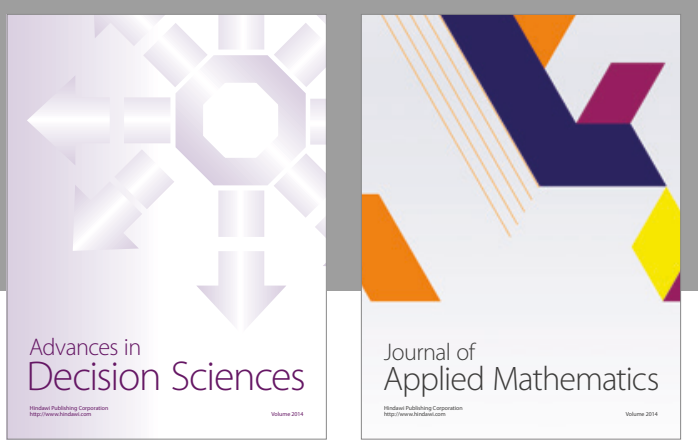

Algebra

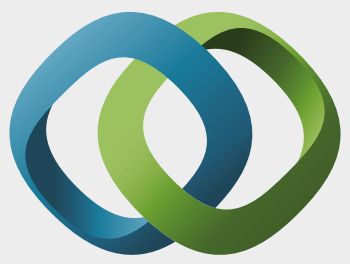

\section{Hindawi}

Submit your manuscripts at

https://www.hindawi.com
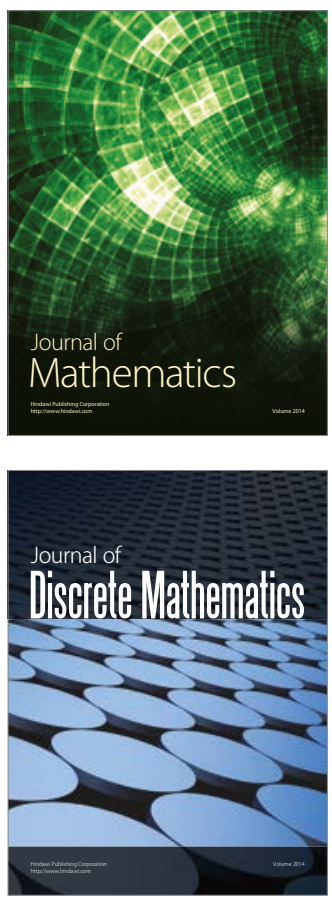

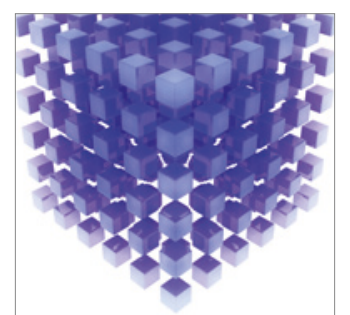

Mathematical Problems in Engineering
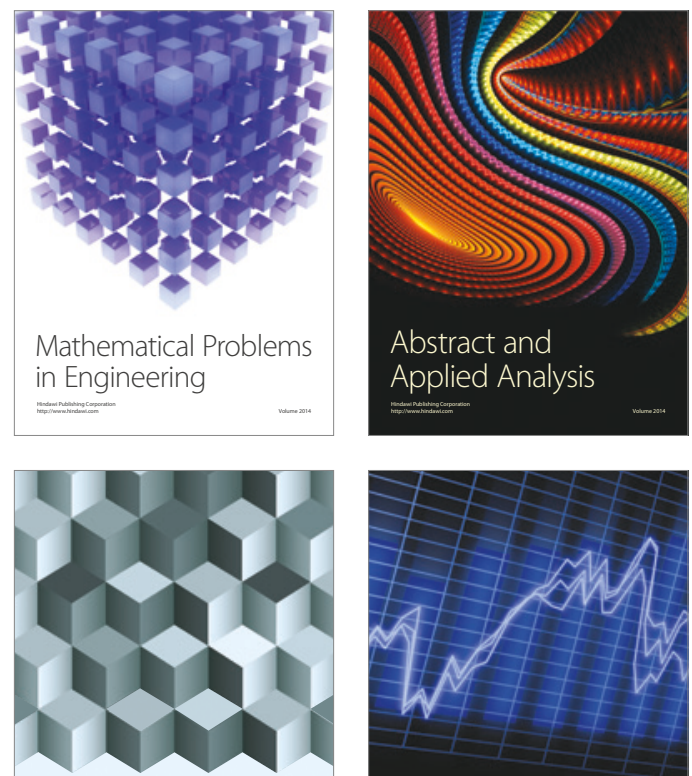

Journal of

Function Spaces

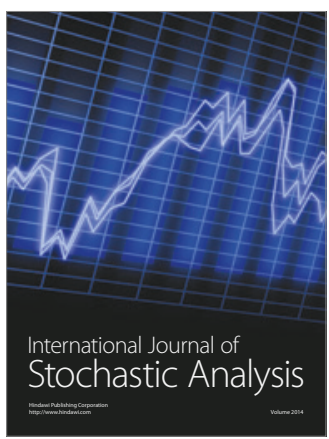

Probability and Statistics
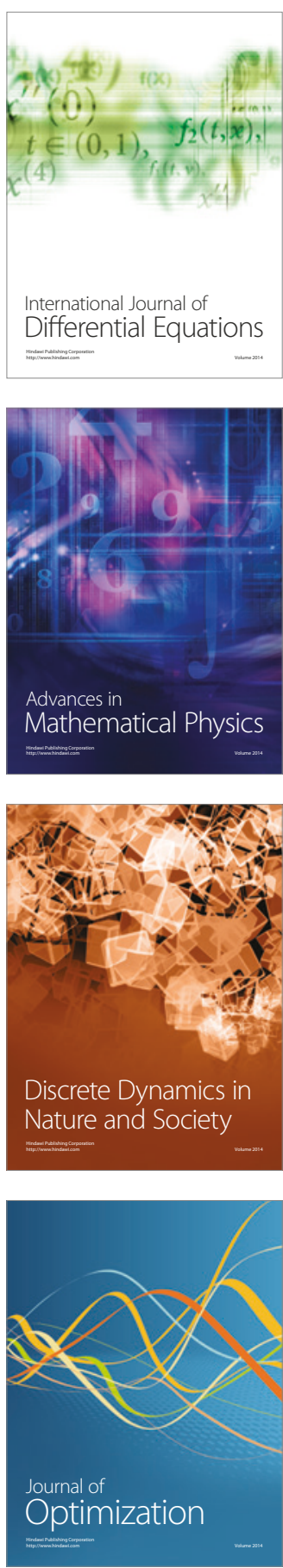